\title{
LA EDUCACIÓN SUPERIOR VIRTUAL Y SU INFLUENCIA EN EL EMPRENDIMIENTO EN COLOMBIA: REVISIÓN DE LITERATURA
}

The influence of higher education on entrepreneurship in Colombia: a review of literature

Sandra Milena Chicas Sierra

smchicas@poligran.edu.co

Institución Universitaria Politécnico Grancolombiano

Colombia

Resumen

El presente artículo de revisión tiene como objetivo determinar el estado del arte de la investigación acerca de la influencia de la educación superior virtual en el emprendimiento en Colombia. El emprendimiento está asociado con el desarrollo económico y social de los países y, a través de la educación presencial se busca fomentar la innovación y el emprendimiento, sin embargo, aún es escasa la investigación acerca del papel de la educación virtual y su influencia en el emprendimiento. Por tal razón, surge la pregunta: ¿hasta dónde ha llegado la investigación acerca de la influencia de la educación virtual en el emprendimiento en Colombia? Para esto, se realizó una revisión de 50 referencias bibliográficas de los últimos 10 años (2010 en adelante) que abordarán estos temas. En la ejecución de la revisión se establecieron los criterios de análisis que incluyeron: desempleo, competencias emprendedoras, calidad de la educación virtual, emprendimiento social, intención emprendedora, perspectiva institucional y promoción de cultura emprendedora. Los resultados muestran que aún no se ha escrito mucho sobre la influencia de la educación virtual en el emprendimiento y que Colombia ha sido el país que más escritos ha publicado sobre el tema.

Palabras clave:

Emprendimiento social, educación virtual, cultura emprendedora, intención emprendedora 


\section{INTRODUCCIÓN}

Este artículo de revisión de literatura es el resultado del análisis bibliográfico de 50 artículos de investigación, cuyo objetivo principal era determinar el estado del arte en la investigación sobre la influencia de la educación superior virtual en el emprendimiento en Colombia. Para esta búsqueda se utilizaron bases de datos como Science Direct, Web of Science, Scielo, Readalyc y Google académico entre los meses de marzo a mayo de 2020. La iniciativa de este proceso de investigación surgió como producto del interés por evaluar la calidad y pertinencia de los currículos de programas virtuales y su influencia en el emprendimiento entre sus estudiantes. De ahí surgió la duda acerca de si realmente la educación virtual influye de manera positiva en el emprendimiento.

En tiempos de pandemia como los que está atravesando el mundo con la pandemia por COVID-19, el emprendimiento se convierte en una herramienta importante para el desarrollo económico de los países, más aún, si se realiza en conjunto con las tecnologías de la información. Es por eso, que la educación superior virtual es clave en el proceso de influir e impactar de manera positiva a los estudiantes, en torno a la generación de emprendimientos sostenibles.

En Colombia la ley 1014 de 2006 tiene como fin promover el emprendimiento a través de las instituciones educativas. Por tal razón, las instituciones de educación superior son las encargadas de fomentar e influir en sus estudiantes la creación de emprendimientos sostenibles, a través de la adaptación de planes académicos y programas educativos que vayan en concordancia con el emprendimiento (Ministerio de Educación Nacional, 2012). Ahora bien ¿qué papel juegan entonces las instituciones de educación superior modalidad virtual? ¿Acaso se ha investigado si tiene influencia sobre el emprendimiento en sus estudiantes?

Ante esta inquietud, el objetivo de esta investigación fue exponer las diferentes relaciones que existen entre los conceptos de emprendimiento y educación superior virtual. Para esto, el trabajo se dividirá en cinco partes. La primera parte abordará la definición de emprendimiento y educación superior virtual. La segunda parte presentará los antecedentes de la investigación; la tercera parte explicará la metodología que se utilizó a través de una revisión bibliográfica de artículos relacionados con el tema, luego de su clasificación y categorización, de acuerdo con los criterios seleccionados. La cuarta parte presentará los resultados del análisis de la literatura investigada. Finalmente se presentarán las conclusiones de esta revisión de literatura.

\section{Concepto de emprendimiento}

El concepto de emprendimiento proviene del francés "entreprende" y del inglés "entrepreneur" que significa aquel que se compromete a organizar, gerenciar y asumir riesgos de un negocio (Uribe \& Reinoso, 2013). Otra definición de emprendimiento es la que expone Schumpeter al definir al emprendedor como aquel que promueve la innovación y realiza nuevas combinaciones en los procesos de producción (Labarcés-Ballestas, 2015). El emprendimiento además está asociado con el desarrollo económico de los países, ya que, entre más emprendimientos tenga un país, mayores serán las oportunidades de que se generen empleos.

La globalización, a su vez, ha contribuido a que más personas piensen en el emprendimiento como una opción para el desarrollo económico y social. De acuerdo con Duarte y Ruíz (2010), las ideas innovadoras causan impacto en lo económico y social en las comunidades, al mismo tiempo que mejoran la calidad de vida de las personas.

El emprendimiento, entonces, está asociado con el crecimiento económico de los países, especialmente el emprendimiento social; tal como lo mencionan Bikse, Rivza, y Riemere (2015) "el emprendedor social sirve como fuerza motriz del desarrollo económico" (p.470).

De acuerdo con un artículo publicado por la revista Semana, el emprendimiento social es una mezcla entre una solución innovadora a los problemas sociales, y al mismo tiempo, una empresa que genera ingresos y 
rentabilidad. El mismo artículo hace énfasis en la importancia que las instituciones de educación superior le están dando al emprendimiento, como herramienta clave para ofrecer programas académicos exitosos (Semana, 2017).

A través del emprendimiento, se favorece el crecimiento de las economías mediante las iniciativas de las empresas que crean empleos y encadenamientos productivos, aumentando la riqueza, los tributos y la competitividad, tanto de las empresas como de las economías (Durán-Peralta, 2019). Para Uribe-Macias (2017), por otra parte, el emprendimiento está definido por un conjunto de competencias específicas que llevan a la construcción o generación de algo nuevo.

El Ministerio de Educación Nacional, en su artículo 1 de la ley 1014 de 2006 afirma que "el emprendimiento es una forma de pensar, razonar y actuar centrada en las oportunidades, que resulta en creación de valor que beneficia a la empresa, la economía y la sociedad” (Ministerio de Educación Nacional, 2012, pág. 1).

Otro concepto de emprendedor es el que exponen Saldarriaga y Guzmán (2018) al señalar que "el emprendedor es aquel que tiene una idea de negocio, hace una inversión de capital y asume riesgos" (p.129). También afirman que los emprendedores deben ser personas creativas, innovadoras y con un alto nivel de automotivación, disciplina y manejo de la frustración. Por otra parte, Rodríguez-Moreno y Gómez- Murillo (2014) señalan que el emprendimiento es un proceso orientado a la creación de una empresa formal que se basa en el desarrollo de una idea innovadora.

Es ahí donde la educación superior juega un papel importante para promover el emprendimiento y la innovación en sus estudiantes, para que éstos desarrollen competencias y habilidades que les permitan crear emprendimientos sostenibles, así lo afirma Monsalve (2010) "fortalecer el emprendimiento es una tarea que debe ser abordada por la educación superior" (p.53). Además, la educación emprendedora aumenta el número de estudiantes con capacidades e intención de crear empresas. Según Barba-Sánchez \& Atienza-Sahuquillo (2018) "existe un efecto positivo en entre la educación emprendedora y la intención de crear empresas" (p.57).

De acuerdo con Duarte y Ruiz (2010) las instituciones de educación deben incluir en sus currículos y planes de estudio la educación emprendedora para desde ahí generar oportunidades laborales y sinergias entre los sectores productivos y las instituciones de educación superior, con el fin de obtener un mayor desarrollo económico y social. Para Guarnizo-Vargas y Velásquez-Moreno (2015) el emprendimiento se puede ver como la posibilidad de fortalecer el sistema educativo, cuyo propósito es contribuir al desarrollo del proyecto de vida de los estudiantes, así como el de la comunidad.

Por otra parte, el Ministerio de Educación Nacional de Colombia indica que la cultura de emprendimiento debe ser incluida en las aulas de clases, y que las instituciones de educación superior deben crear los ambientes apropiados para que los estudiantes desarrollen capacidades emprendedoras (Ministerio de Educación Nacional, 2012).

\section{Educación superior Virtual en Colombia}

La globalización trajo consigo un nuevo fenómeno asociado a la educación: la virtualización o educación online. Tal como lo explica Chan (2016) "la virtualización de la educación se presenta como un fenómeno en donde se actualizan los métodos educativos con el uso de TICs" (p.3). Con el avance en las TIC son cada vez más las instituciones de educación superior que ofrecen programas virtuales de pregrado y posgrado, y por tanto, más personas tienen acceso al conocimiento y a oportunidades de creación de empresas.

Arias, Fernández y Ramírez (2016) explican que la relación entre la educación virtual y el emprendimiento es muy estrecha, ya que a través de la educación virtual los estudiantes aprenden y adquieren competencias que les permiten ser innovadores y creadores de empresas. En el mundo tan complejo en el que se encuentra la sociedad 
actual y, con las altas demandas de la sociedad del conocimiento y la revolución 4.0, se necesitan más estudiantes creativos, emprendedores e innovadores.

Otro punto importante para tener en cuenta es que muchos de los emprendimientos de hoy en día son emprendimientos virtuales o emprendimientos que utilizan el internet y las diferentes tecnologías de la información para el desarrollo de los negocios (Arias \& Castillo, 2011). En especial lo relacionado con el emprendimiento social, así lo explican Morales-Gutiérrez, Ariza-Montes, y Muñiz-Rodríguez (2012) que el fenómeno del emprendimiento social se ha empoderado cada vez más gracias al desarrollo de nuevas tecnologías y a las redes sociales.

La implementación de las TIC en la educación superior ayuda a que los estudiantes tengan más posibilidades de ser emprendedores sociales y contribuir con problemas asociados con la exclusión, el desempleo y el daño medio ambiental, entre otros, creando bienes y servicios innovadores y digitales que generen valor a los consumidores (Santos, Barroso, \& Guzmán, 2013). Khajeh-Dangolani (2011) expone, además, que el éxito de un emprendimiento está asociado, no solo con la creatividad y la innovación, sino con el uso apropiado de las tecnologías de la información; es decir que existe una relación directa y positiva entre el emprendimiento y el uso de las herramientas digitales.

En ese sentido, la educación virtual se presenta como una herramienta útil para aquellas personas que, por diferentes razones, no pueden acceder a la educación superior de manera presencial. Por tal razón, los ambientes virtuales de aprendizaje son espacios de orientación pedagógica, apoyados en las tecnologías de la información, para facilitar la comunicación entre docentes y estudiantes (Reinoso \& Sánchez, 2017).

La educación virtual se entiende como el uso del ciberespacio para desarrollar programas de educación, y, aunque no requiere un encuentro presencial con el docente, permite establecer una relación de enseñanza- aprendizaje, a través del uso de herramientas digitales (Rodríguez, Lorduy, \& Ariza, 2014). A través de la educación superior virtual se pueden incorporar materiales de estudio asociados con marketing digital, creación de páginas web, ecommerce, branding y hasta uso de redes sociales para promocionar los emprendimientos.

Cada vez es más relevante el papel de la educación superior en torno a incentivar el emprendimiento, creando una sociedad más innovadora y que sea capaz de aprovechar las oportunidades del entorno, especialmente en materia de tecnologías de la información y las comunicaciones. Emprendimientos como Facebook, por ejemplo, fueron creados por estudiantes universitarios, que hicieron buen uso de la tecnología que tenían a su alcance. Microsoft y Apple son otros ejemplos claros de cómo, a través de las aulas de clase, los estudiantes pueden desarrollar emprendimientos exitosos de la mano de las tecnologías de la información y las comunicaciones.

Las instituciones de educación superior tienen un rol influyente dentro de sus propuestas de programas para contribuir a formar ciudadanos más emprendedores (Salmerón-Gómez \& Gómez-Haro, 2012). Mivehchi (2019) señala que el entorno de las tecnologías de la información es una nueva puerta que aumenta las posibilidades para el emprendimiento, porque brinda las herramientas para la creación de negocios virtuales, tiendas en línea y mayor acceso a mercados.

Según un informe del Global Entrepreneurship Monitor, la creación de empresas depende, en gran medida, del nivel educativo de los emprendedores. Esto deja claro el rol tan decisivo que juegan las universidades en la promoción de la cultura emprendedora y el desarrollo económico y social del país (Correales, 2013).

Colombia no es ajena al tema de educación virtual, tanto que ya son varias las universidades que ofrecen programas de pregrado y posgrado bajo esta nueva modalidad. Por eso, se hace importante investigar si estas universidades han realizado estudios o publicaciones acerca de la incorporación de las TIC en sus programas virtuales y su influencia en el emprendimiento; especialmente, porque en la actualidad, dadas las circunstancias 
de la pandemia del COVID-19, la educación, en todos sus niveles, ha migrado al modelo virtual y el emprendimiento se convierte en una herramienta útil para que las personas se reinventen y desarrollen ideas y negocios sostenibles y digitales.

La ley 30 de 1992 establece el marco regulatorio de la educación superior en Colombia en donde especifica que los estudiantes deben recibir una educación superior que los incentive a ser autónomos, creativos e independientes; la ley 115 por su parte, establece que las instituciones de educación superior deben ofrecer programas orientados al desarrollo de la capacidad científica y tecnológica, con el fin de mejorar la calidad de vida de las personas (Camargo-Fonseca, 2012). A su vez, el decreto 1295 de 2010 define la educación virtual y a distancia como aquella que cuenta con una metodología y estrategias de enseñanza-aprendizaje que permiten a los estudiantes y docentes romper las barreras de espacio y tiempo; promoviendo en el estudiante el aprendizaje autónomo, la constancia y la autodisciplina (Cháves-Torres, 2017).

La formación en educación superior, entonces, debe garantizar pertinencia y calidad en sus programas virtuales para que los estudiantes se conviertan en generadores de ideas con valor agregado con sentido social y económico (Alvardo-Muñoz \& Rivera-Martínez, 2011).

\section{REVISIÓN DE LITERATURA}

La literatura revisada presenta como principales hallazgos que existe una relación directa entre la educación superior y el emprendimiento, especialmente en la sociedad colombiana que se ha caracterizado por desigualdades sociales y económicas. La Universidad es el primer paso para la articulación y el desarrollo de las propuestas de emprendimiento.

Sánchez-García, Ward, Hernández y Florez (2017), por ejemplo, señalan que la educación superior tiene un impacto positivo en el emprendimiento porque desarrolla en los estudiantes competencias asociadas a la toma de decisiones, trabajo en equipo, liderazgo, análisis y solución de problemas, creatividad, innovación y comunicación asertiva.

En el tema de la educación superior virtual, la literatura encontrada de Arias, Fernández y Ramirez (2016) sugiere, además, que esta influye de manera positiva en el emprendimiento ya que promueve en el estudiante el uso de herramientas tecnológicas, el trabajo autónomo y colaborativo, la innovación y la investigación; actividades que caracterizan a un emprendedor.

Para Freire (2016) el emprendimiento se puede promover desde la enseñanza en instituciones de educación superior, a través de programas de acompañamiento y asesoría en la planificación de proyectos innovadores. Herramientas virtuales como las redes sociales pueden incorporarse en la educación superior para desarrollar competencias emprendedoras en los estudiantes, contribuyendo a la creación de empresas de base tecnológica (Morales \& Corredor, 2016).

Khajeh-Dangolania (2011) sostiene que la educación superior virtual está asociada con el emprendimiento porque el éxito de un proyecto emprendedor se define, en gran parte por la implementación adecuada de las tecnologías de la información, combinada con las competencias adquiridas desde la institución educativa como manejo de riesgo, proactividad e innovación.

A través de la educación virtual, Colombia ha iniciado el fortalecimiento del emprendimiento y la generación de cultura emprendedora incluyendo, como parte de sus planes académicos, aspectos asociados con asesorías en línea, socialización en el uso de herramientas digitales y comunicación virtual (Ramos-Rubio, 2017). 


\section{Metodología de la Revisión bibliográfica}

La metodología utilizada para este artículo es de tipo cualitativa con una técnica de investigación documental, ya que se realizó un análisis del estado del arte relacionado con los temas de educación virtual y emprendimiento en Colombia. Los artículos y documentos objeto de análisis fueron sacados de bases de datos como Google Scholar, Science Direct, Scielo, Web of Science y Redalyc. Para esta investigación se implementaron fórmulas de búsqueda como “Educación virtual y Emprendimiento en Colombia”, "Virtual Entrepreneurship”, “Virtual AND Higher Education AND Entrepreneurship" o “Entrepreneurship AND Colombia”. Otro criterio de búsqueda aplicado fue la delimitación por año (2010 en adelante), así como la indexación, especialmente para la búsqueda en Google académico, donde se puede encontrar desde artículos de revistas indexadas hasta tesis de pregrado o maestría y resúmenes de congresos. Luego de aplicar estas fórmulas el resultado fue seleccionar un total de 50 artículos que hacían énfasis en temas de educación virtual y emprendimiento.

Una vez terminada la búsqueda y selección de los artículos, el siguiente paso fue elaborar el estado del arte. Para eso se desarrolló un cuadro de Excel que organizó la información de la siguiente manera: título, resumen, palabras clave, año de publicación, país de publicación, género del autor y la referencia bibliográfica. Adicionalmente, se establecieron unos temas clave de clasificación de los artículos que fueron: desempleo, competencias emprendedoras, calidad de la educación virtual, emprendimiento social, intención emprendedora, perspectiva institucional y promoción de cultura emprendedora. Posteriormente, se realizó el análisis detallado de cada aspecto por separado, para identificar cuántos artículos se habían publicado de cada uno. Se realizó un Excel por cada aspecto (año de publicación, género del autor, país de publicación y por cada criterio seleccionado), luego, se realizaron gráficas con la información ya clasificada.

\section{RESULTADOS Y ANÁLISIS}

Los resultados de la revisión bibliográfica se dieron a partir de 50 artículos de investigación, de los cuales se analizaron cuatro aspectos importantes como son el año de publicación de los artículos, el género del autor (hombre o mujer), las temáticas clave (desempleo, competencias emprendedoras, calidad de la educación virtual, emprendimiento social, intención emprendedora, perspectiva institucional y promoción de cultura emprendedora) y los países que más publicaciones realizaron en relación con los temas investigados que fueron educación virtual y emprendimiento.

El primer análisis que se realizó fue el año de publicación de los artículos, en donde se pudo evidenciar que los años en los que más artículos se publicaron sobre educación virtual y emprendimiento fueron en el 2012 y 2014 con 7 artículos cada uno. Sin embargo, la tendencia desde el 2010 que fue el primer año analizado, fue creciente hasta el 2014 donde luego se presentó una disminución que se ha mantenido hasta el presente año (2020), tal como lo evidencia la Gráfica 1. 


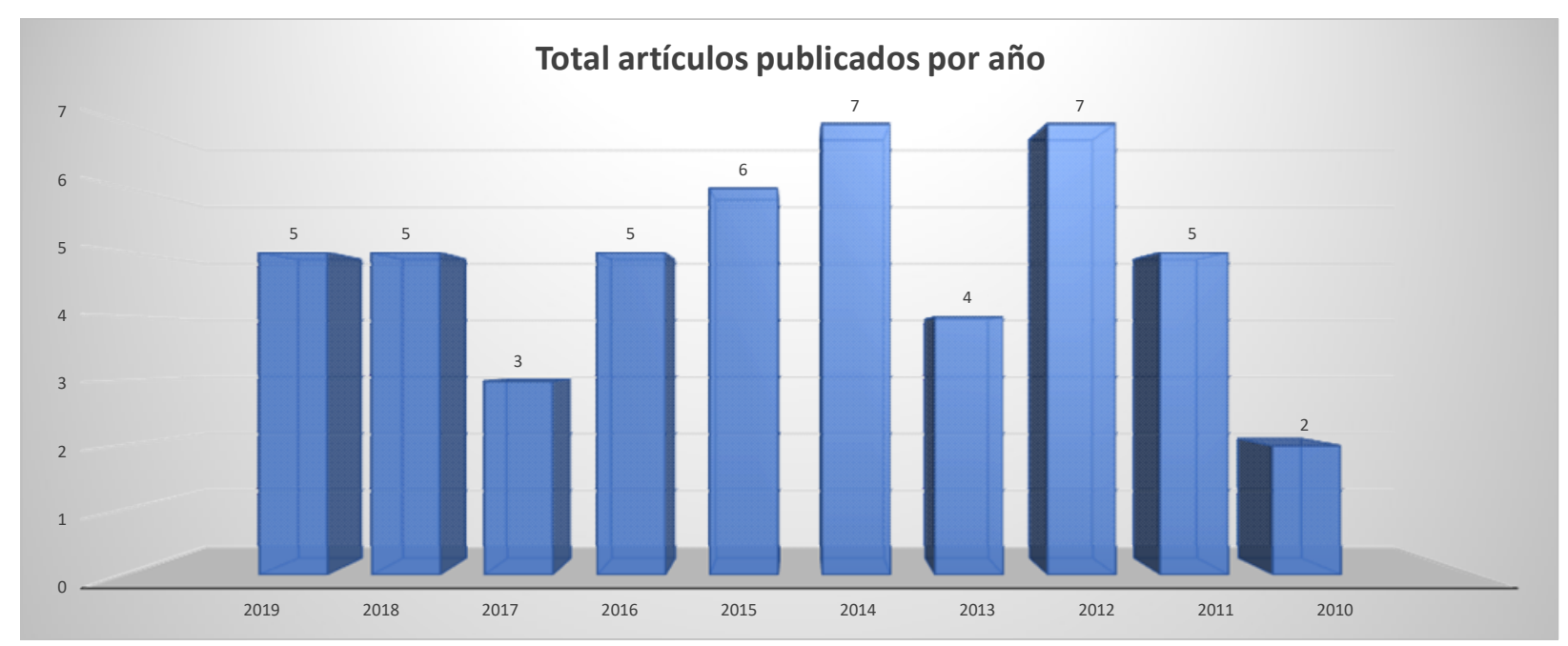

Gráfica 1: Total artículos publicados por año. Fuente: Elaboración propia

El segundo análisis que se desarrolló fue el de los países que más publicaciones habían realizado sobre educación virtual y emprendimiento. En este análisis se encontró que Colombia fue el país que realizó el mayor número de publicaciones en el periodo de tiempo comprendido entre 2010-2019, con un total de 23 artículos relacionados con la educación virtual y el emprendimiento. Luego le sigue España con 9 publicaciones de artículos en el mismo periodo de tiempo. Los demás países tuvieron entre 1 y 3 publicaciones durante el periodo de tiempo analizado. Esto se puede evidenciar en la gráfica 2.

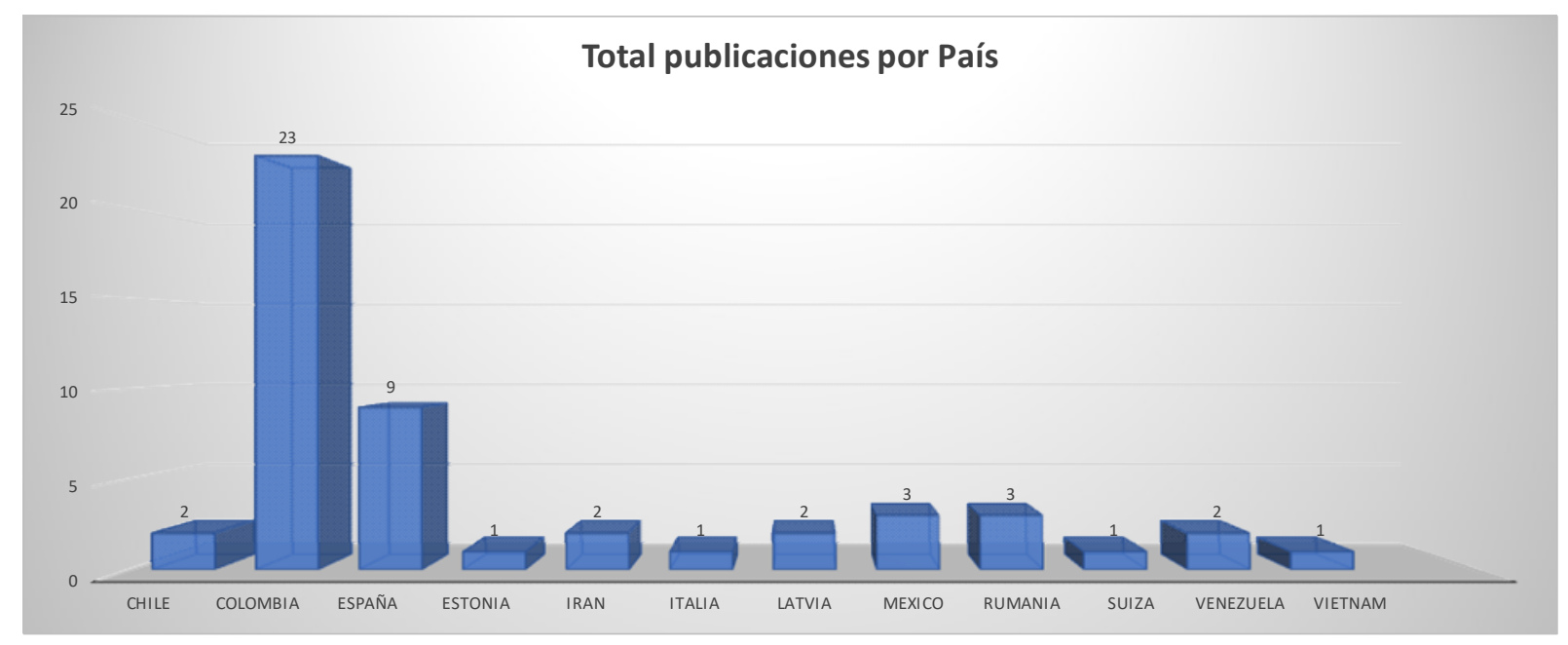

Gráfica 2: Total publicaciones de artículos por país. Fuente: Elaboración propia

El tercer análisis que se realizó de la revisión bibliográfica fue el género del autor para identificar quién había escrito más sobre educación virtual y emprendimiento, los hombres o las mujeres. El resultado arrojó que los hombres habían escrito 18 de los 50 artículos analizados, mientras que las mujeres escribieron 16 de los 50 artículos analizados y, los 16 artículos restantes fueron escritos en conjunto entre hombres y mujeres, tal como se evidencia en la gráfica 3. 


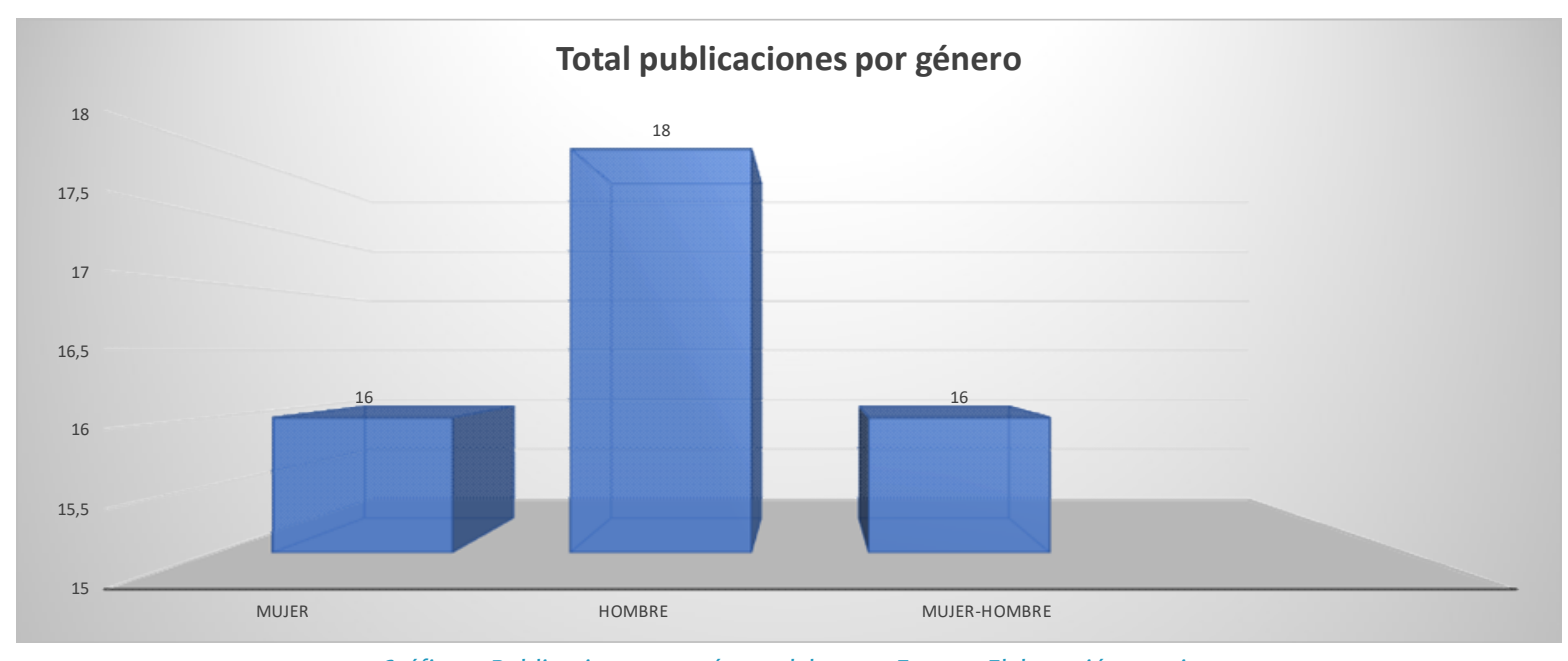

Gráfica 3: Publicaciones por género del autor. Fuente: Elaboración propia

Finalmente, con el análisis de los criterios o categorías asociadas con emprendimiento y educación virtual se pudo visualizar que los artículos que más se publicaron fueron acerca de intención emprendedora y emprendimiento social con 9 artículos en total, seguido de competencias emprendedoras con 8 artículos; mientras que el criterio de promoción de cultura emprendedora tuvo 7 artículos escritos y los criterios asociados con perspectiva institucional, cultura de emprendimiento y calidad de la educación virtual tuvieron 5 publicaciones cada uno. Por su parte, el criterio de desempleo solo contó con 2 publicaciones en el periodo de tiempo investigado. Esto se puede evidenciar en la gráfica 4.

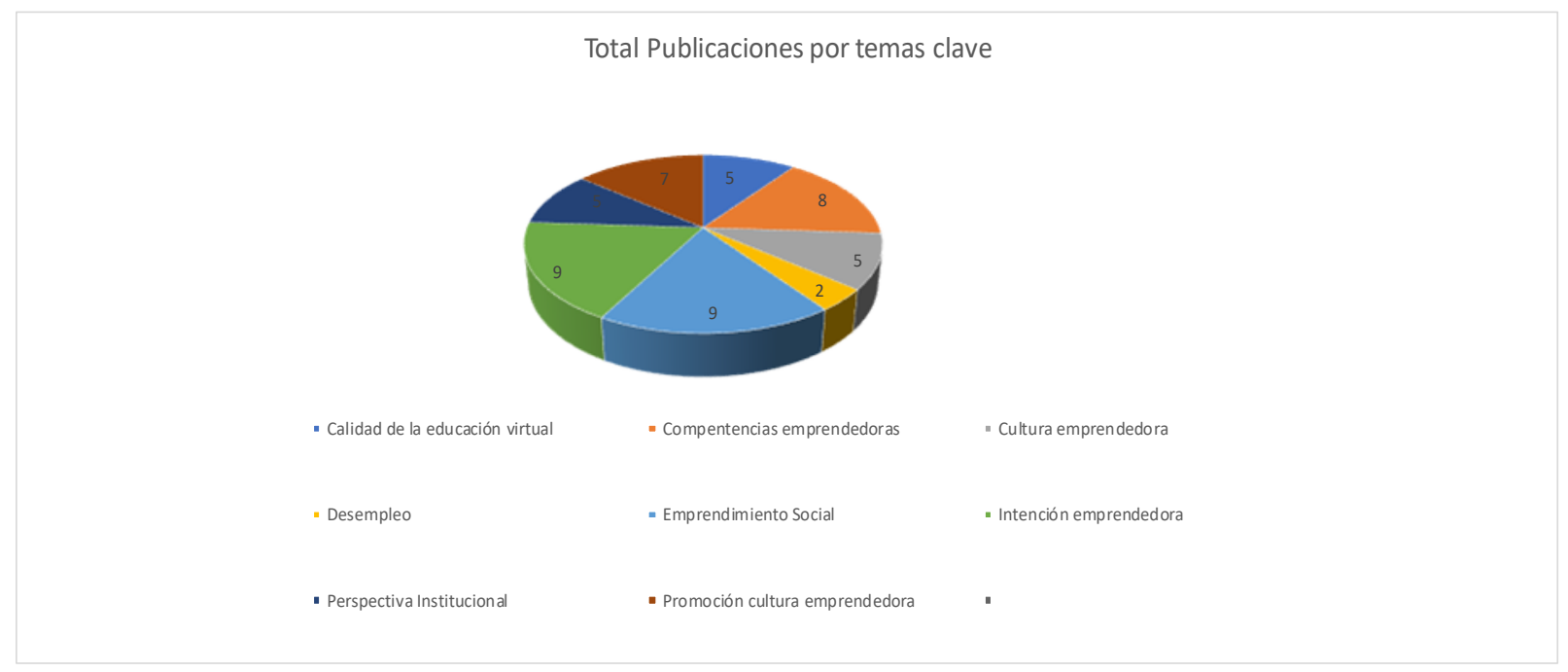

Gráfica 4: Total publicaciones por criterios. Fuente: Elaboración propia

\section{Discusión}

El emprendimiento es una herramienta útil para la generación de empleos en las economías, así como también contribuye a desarrollar competencias gerenciales como el liderazgo, el trabajo en equipo, análisis de datos y toma de decisiones, entre otros. Por eso, la educación es una aliada en el proceso de formación de emprendedores, especialmente en emprendimientos con apoyo de las TIC. Con la llegada de la cuarta revolución 
industrial, la educación virtual cada vez toma más fuerza en el mundo y, en Colombia, son cada vez más las universidades que le apuestan a programas de pregrado y posgrado bajo la modalidad virtual.

En un mundo tan globalizado, el uso de las herramientas digitales para la creación de emprendimientos sostenibles amerita un modelo educativo enmarcado desde la virtualidad, que forme a los estudiantes no solo en el uso de estrategias y herramientas digitales, sino en competencias y habilidades emprendedoras.

En ese sentido, se propone ampliar las investigaciones en torno a la importancia de la incorporación de la educación emprendedora en las aulas de clase, a través de los ambientes virtuales de aprendizajes. De esa manera, las instituciones de educación superior en Colombia tendrían la necesidad de modificar sus planes educativos y sus programas académicos virtuales, para ser promotores de la cultura emprendedora. El emprendimiento social enseñado desde las aulas de clase y con ayuda de la tecnología es otro tema del que se debe realizar mayor investigación, ya que, a través de emprendimientos sociales, los estudiantes no solo llegan a conocer la realidad de las comunidades, sino que proponen bienes y servicios innovadores que mejoren las condiciones y la calidad de vida de esas comunidades.

\section{Conclusión}

Este artículo de revisión bibliográfica se dio con la intención de indagar sobre la importancia de la educación superior virtual en el emprendimiento en Colombia, a través del análisis de artículos publicados en los últimos diez años. El objetivo era desarrollar un estado del arte acerca de la influencia positiva que tiene la educación superior modalidad virtual en el emprendimiento. Se procedió, entonces, a realizar la revisión bibliográfica de 50 artículos de investigación para poder identificar si la educación superior virtual ejerce alguna influencia sobre el emprendimiento.

De acuerdo con el análisis bibliográfico realizado, se puede concluir que la educación superior y el emprendimiento tienen una relación directa; y que la educación superior virtual tiene un rol preponderante en la promoción de la cultura emprendedora. Sin embargo, se evidencia que aún es poca la información que se tiene y que se ha escrito o investigado acerca de la importancia de promover el emprendimiento a través de la educación superior virtual. Ahora bien, es importante resaltar que varios países han empezado a escribir más artículos sobre educación virtual y emprendimiento, en el periodo comprendido entre 2010 y 2019, que fue el periodo analizado. Esto hace pensar que hay un alto grado de interés en continuar investigando acerca de la influencia que ejerce la educación superior virtual en el emprendimiento. Especialmente porque la tecnología cada vez más se está incorporando en la educación como herramienta para la adquisición de competencias gerenciales. Además, hoy en día los emprendimientos digitales están en aumento en el mundo, ya que se pueden crear negocios sostenibles a través de redes sociales como Facebook o Instagram, blogs, ambientes virtuales de aprendizaje y hasta juegos virtuales.

Las instituciones de educación superior juegan un papel importante a la hora de enseñar y promover el emprendimiento en sus estudiantes, y más ahora con el uso de las TIC en la educación, la virtualidad también se convierte en un modelo de enseñanza aprendizaje de cultura emprendedora y emprendimientos sociales. Sin embargo, los resultados de esta revisión bibliográfica señalan que no es mucho lo que se ha escrito sobre cultura emprendedora y la perspectiva institucional.

Frente al tema de emprendimiento asociado con el desempleo, que en muchos de los casos es la razón principal para iniciar un negocio, tampoco se ha realizado mayor investigación y, por consiguiente, es muy poco lo que se ha escrito acerca del desempleo como motivación para emprender desde la academia. Actualmente, el mundo está requiriendo mayor investigación relacionada con el emprendimiento digital y el uso de herramientas virtuales para promover el emprendimiento; de esta manera, se contribuye a generar los empleos que se perdieron debido a la pandemia mundial del COVID-19. 
La educación para el emprendimiento es una herramienta importante que todas las universidades deberían implementar en sus aulas de clase. Se debería medir más la intención emprendedora de los estudiantes colombianos, su capacidad para emprender y sus conocimientos en creación de empresas sostenibles; así como también medir la calidad de la educación virtual asociada con el emprendimiento. De igual manera, el gobierno debe apoyar las iniciativas de emprendimiento en las instituciones de educación superior y fomentar la cultura emprendedora como parte del proceso de enseñanza-aprendizaje e invertir en conocimiento, investigación y desarrollo. De esa manera, se podrán realizar más investigaciones y publicaciones relacionadas con la influencia de la educación superior modalidad virtual en el emprendimiento.

Finalmente, las instituciones de educación superior deben promover que sus estudiantes y docentes investiguen y escriban más acerca de la importancia y la influencia que tiene la enseñanza del emprendimiento, especialmente para los estudiantes de modalidad virtual, ya que son ellos los que pertenecen, en la mayoría de los casos a poblaciones excluidas; esto les brindará oportunidades de empleo y desarrollo económico y social en sus regiones.

\section{Referencias bibliográficas}

Alvardo-Muñoz, O., \& Rivera-Martínez, W. (2011). Universidad y emprendimiento, aportes para la formación de profesionales emprendedores. Cuadernos de Administración, 61-74.

Arias, A., Fernández, L., \& Ramírez, O. (2016). El emprendimiento en la educación virtual, una alternativa para incitar la creación de empresa, la innovación y la realización personal. virtualmente , 1-12.

Arias, C. M., \& Castillo, E. (2011). La educación para el emprendimiento y empresarismo virtual: potencialidades. Revista virtual Universidad Católica del Norte, 1-8.

Barba-Sánchez, V., \& Atienza-Sahuquillo, C. (2018). Entrepreneurial intention among engineering students: The role of entrepreneurship education. European Research on management and business economics, 53-61. doi:http://dx.doi.org/10.1016/j.iedeen.2017.04.001

Bikse, V., Rivza, B., \& Riemere, I. (2015). The Social Entrepreneur as a Promoter of Social Advancement. Procedia social and behavioral sciences, 469-478. doi:doi: 10.1016/j.sbspro.2015.03.405

Camargo-Fonseca, P. E. (2012). Importancia del emprendimiento en el ámbito educativo. Emprendimiento, 5060.

Chan, M. E. (2016). La virtualización de la educación superior en América Latina: entre tendencias y paradigmas. Revista de Educación a Distancia , 1-32.

Cháves-Torres, A. (2017). La educación a distancia como respuesta a las necesidades del siglo XXI. Revista Academia y Virtualidad, 23-41. doi:http://dx.doi.org/10.18359/ravi.2241

Correales, J. P. (07 de 31 de 2013). Dinero. Obtenido de https://www.dinero.com/opinion/columnistas/articulo/emprendimiento-nace-aulas/180924

Duarte, T., \& Ruiz, M. (2010). Emprendimiento, una opción para el desarrollo. Scientia et Technica, 326-331.

Duarte, T., \& Ruiz, M. (2010). Emprendimiento, una opciòn para el desarrollo. Scientia et Technica, 326-331.

Durán-Peralta, J. (2019). Desarrollo regional y emprendimiento: Evidencia para Colombia. El trimestre económico, 467-490. doi:doi: 10.20430/ete.v86i342.656 
Freire, M. T. (2016). Educación en emprendimiento. Revisión de la literatura económica. Iberian Journal of the History of Economic Thought, 82-100. doi:http://dx.doi.org/10.5209/rev_IJHE.2015.v2.n2.52052

Guarnizo-Vargas, C. M., \& Velásquez-Moreno, J. R. (2015). La cultura del emprendimiento y la empresarialidad en instituciones educativas de Colombia: realidades y oportunidades. Logos Ciencia y Tecnología, 227-234.

Khajeh-Dangolania, S. (2011). The Effect of Information Technology in the Entrepreneurship: A Case Study in Golestan Province IRAN. Procedia - Social and Behavioral Sciences, 10-12.

Labarcés-Ballestas, C. (2015). Aspectos de Innovación Schumpeteriano: El Eemprendimiento, el perfildel empresarioen el contexto social. Dictamen Libre, 73-80.

Ministerio de Educación Nacional. (2012). La cultura del emprendimiento en los establecimientos educativos. Bogotá: Panamericana.

Mivehchi, L. (2019). The role of information technology in woman entrepreneurship. Procedia Computer Science, 508-512.

Monsalve, B. (2010). El emprendimiento y la universidad . Pensamiento americano, 51-54.

Morales, A., \& Corredor, H. (2016). Las redes sociales: una estrategia pedagógica para incentivar el emprendimiento. Ciencia y poder Aéreo, 242-255. doi:Doi: http://dx.doi.org/10.18667/cienciaypoderaereo.522

Morales-Gutiérrez, A., Ariza-Montes, A., \& Muñiz-Rodríguez, N. (2012). El emprendedor social y el eempowerment de las redes sociales. Revista de economía pública, social y cooperativa, 152-177.

Ramos-Rubio, P. (2017). Promoción del Emprendimiento para el Desarrollo Educativo. Revista de la Facultad de Contaduría y Ciencias Administrativas, Vol. 2 Núm. 3, 18-36.

Rodríguez, G., Lorduy, V., \& Ariza, M. (2014). Calidad de la Educación Superior a distancia y virtual: Un análisis de desempeño académico en Colombia. Investigación y Desarrollo, 58-99.

Rodríguez-Moreno, D. C., \& Gómez-Murillo, A. X. (2014). Las competencias emprendedoras en el departamento de Boyacá. Revista Apuntes del CENES, 217-242.

Saldarriaga, M. E., \& Guzmán, M. F. (2018). Enseñanza del emprendimiento en la educación superior: ¿Metodología o modelo? Revista EAN, 125-142. doi:DOI: https://doi.org/10.21158/01208160.n85.2018.2054

Salmerón-Gómez, R., \& Gómez-Haro, S. (2012). Relación entre los factores institucionales y el emprendimiento: análisis mediante técnicas cuantitativas. Revista de Métodos Cuantitativos para la Economía y la Empresa, 54-72.

Sánchez-García, J. C., Ward, A., Hernández, B., \& Florez, J. L. (2017). Educación emprendedora: Estado del arte. Propósitos y Representaciones, 401-473. doi:http://dx.doi.org/10.20511/pyr2017.v5n2.190

Santos, F., Barroso, M. d., \& Guzmán, C. (2013). La economía global y los emprendimientos sociales. Revista de economía mundial, 177-196.

Semana . (03 de 07 de 2017). Semana. Obtenido de https://www.semana.com/educacion/articulo/que-esemprendimiento-social/517782 
Uribe, M. E., \& Reinoso, J. F. (2013). Emprendimiento y empresarismo. Diferencias, conceptos, cultura emprendedora, idea y proyecto de empresa. Bogota: Ediciones de la U.

Uribe-Macias, M. (2017). El emprendimiento: algunas reflexiones desde un enfoque de revisión. Clío América, 11(22), 219-239. doi:http://10.21676/23897848.2443 